\title{
Surgical resection of superior pulmonary sulcus tumor after neoadjuvant chemoradiation via the anterior transmanubrial approach: a case report
}

\author{
Feichao Bao ${ }^{1}$, Fenghao $\mathrm{Yu}^{1}$, Xiuxiu $\mathrm{Hao}^{1}$, Zhitao Gu${ }^{1}$, Seong Yong Park ${ }^{2}$, Francesco Grossi ${ }^{3}$, \\ Wentao Fang' \\ ${ }^{1}$ Department of Thoracic Surgery, Shanghai Chest Hospital, Shanghai Jiao Tong University, Shanghai, China; ${ }^{2}$ Department of Thoracic and \\ Cardiovascular Surgery, Yonsei University College of Medicine, Seoul, Korea; ${ }^{3}$ Medical Oncology Unit, Department of Medicine and Surgery, \\ University of Insubria, ASST dei Sette Laghi, Varese, Italy \\ Correspondence to: Wentao Fang. Department of Thoracic Surgery, Shanghai Chest Hospital, Shanghai Jiao Tong University, Shanghai 200000, \\ China. Email: vwtfang@hotmail.com.
}

\begin{abstract}
Superior pulmonary sulcus tumor is a cancer arising in the apex of the lung that with potential invasion of the brachial plexus, upper ribs, vertebrae, subclavian vessels, and stellate ganglion. Induction concurrent chemoradiotherapy followed by radical surgical resection with lobectomy combined with any structures in the thoracic inlet invaded by tumor and thorough mediastinal lymph node dissection is the preferred treatment. Both anterior and posterior approaches are applied for resection. Here, we report a 61-year-old man with an $8.6 \mathrm{~cm} \times 5.1 \mathrm{~cm}$ mass arising from the right upper lobe invading the apex of the chest wall. Brachial plexus magnetic resonance imaging suggested tumor invasion of the inferior trunk of the brachial plexus, anterior portion of the first 2 ribs, and suspicious involvement of the subclavian artery. Biopsy of the mass showed stage cT4N2M0, IIIB, poorly differentiated adenocarcinoma. The patient was treated by induction concurrent chemoradiotherapy, which was followed by surgical resection of the right upper lobe and the affected chest wall via the transmanubrial approach. The patient suffered prolonged postoperative air leak and empyema. After continuous chest tube drainage and intrapleural fibrinolytic therapy, he recovered well and was discharged safely. Final pathology showed no viable residue tumor, pathologic complete response of the tumor to induction treatment, a tumor size of $4.1 \mathrm{~cm}$, and no lymph nodes; therefore, the final stage was ypT0NOM0. The transmanubrial approach is feasible for resection of tumor invading the branches of the subclavian artery; however, postoperative empyema which might have resulted from prolonged air leak should be carefully treated by meticulous air leak management.
\end{abstract}

Keywords: Pancoast tumor; superior pulmonary sulcus tumor; multidisciplinary treatment; air leak; case report

Submitted Jun 08, 2021. Accepted for publication Sep 27, 2021.

doi: $10.21037 /$ atm-21-4698

View this article at: https://dx.doi.org/10.21037/atm-21-4698

\section{Introduction}

Superior pulmonary sulcus tumor, also known as pancoast tumor, is a cancer arising in the apex of the lung that with potential invasion of the brachial plexus, upper ribs, vertebrae, subclavian vessels, and stellate ganglion, resulting in characteristic symptoms, like arm and shoulder pain or Horner's syndrome $(1,2)$. The goal of surgical resection is complete resection of the tumor and affected area of the. chest wall. Various surgical approaches, including both the anterior and posterior approach, are applied to expose the tumor and the involved structures and can be personalized depending on the location of tumor and surgeon preference (3). Here, we report a 61-year-old man with lung cancer invading the apex of the chest wall who 

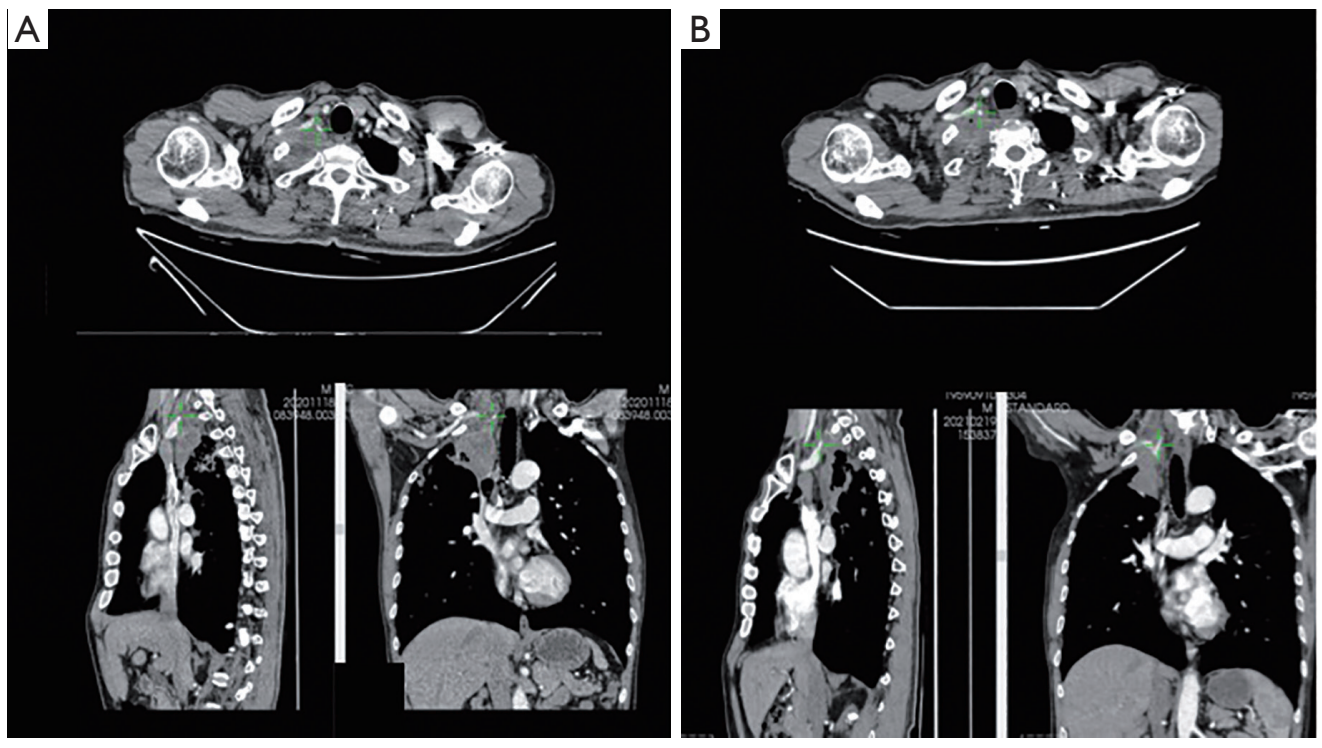

Figure 1 Computed tomography (CT) revealed a mass arising from the right upper lobe invading the thoracic inlet. (A) Images before concurrent chemoradiotherapy; (B) images after concurrent chemoradiotherapy.

was successfully treated with neoadjuvant concurrent chemoradiotherapy followed by surgical resection of the right upper lobe and affected chest wall. We present the following case in accordance with the CARE reporting checklist (available at https://dx.doi.org/10.21037/atm-214698).

\section{Case presentation}

A 61-year-old male smoker was referred for hemoptysis with right arm pain. Computed tomography (CT) revealed an $8.6 \mathrm{~cm} \times 5.1 \mathrm{~cm}$ mass of the right upper lobe invading the thoracic inlet (Figure 1). Positron emission tomographycomputed tomography (PET-CT) showed intense high metabolism of the mass, the ${ }^{18} \mathrm{~F}$-fluorodeoxyglucose (FDG) standard uptake value (SUV)max was 20.3, and the right hilar and mediastinal lymph nodes (station $2 \mathrm{R}$ and $4 \mathrm{R}$ ) were also slightly increased, with an FDG SUVmax of 6.1. For the purpose of confirming the relationship between the mass and the apex of the chest wall, brachial plexus magnetic resonance imaging (MRI) was performed, which suggested tumor invasion of the inferior trunk of brachial plexus, anterior portion of the first 2 ribs, and suspicious involvement of the subclavian artery (Figure 2). However, the intervertebral foramina and vertebral bodies were not invaded. Endobronchial ultrasound-guided transbronchial needle aspiration (EBUS-TBNA) was conducted, and biopsy of the mass showed poorly differentiated adenocarcinoma; therefore, the patient was diagnosed with stage cT4N2M0, IIIB lung cancer. After detailed evaluation and multidisciplinary discussion, induction concurrent chemoradiation was administered. After 50 Gy radiation and 2 cycles of chemotherapy with $880 \mathrm{mg}$ pemetrexed plus $44 \mathrm{mg}$ cisplatin, the arm pain of the patient was relieved, and the patient was reassessed for surgical resection. CT and MRI showed that both the tumor and mediastinal lymph node had shrunk and that the compression of the tumor to the inferior trunk of the brachial plexus had been relieved (Figures 1,2). The patient was diagnosed with stage ycT3N2M0, IIIB lung cancer after induction treatment, surgical resection of the superior pulmonary sulcus tumor was proposed.

A transmanubrial approach without clavicle resection, modified from the classical Dartevelle technique, was adopted for resection (4). The patient was placed in the supine position with his head turned to the left side. An L-shaped incision was made along the anterior border of the sternocleidomastoid muscle which extended inferiorly along the manubrium before turning laterally to a course parallel to the bed of the second intercostal space up to the deltopectoral groove. The manubrium was divided at the midline, and the division was extended into the second intercostal space. After division of the first 2 costal cartilages and internal mammary artery, the osteomuscular 

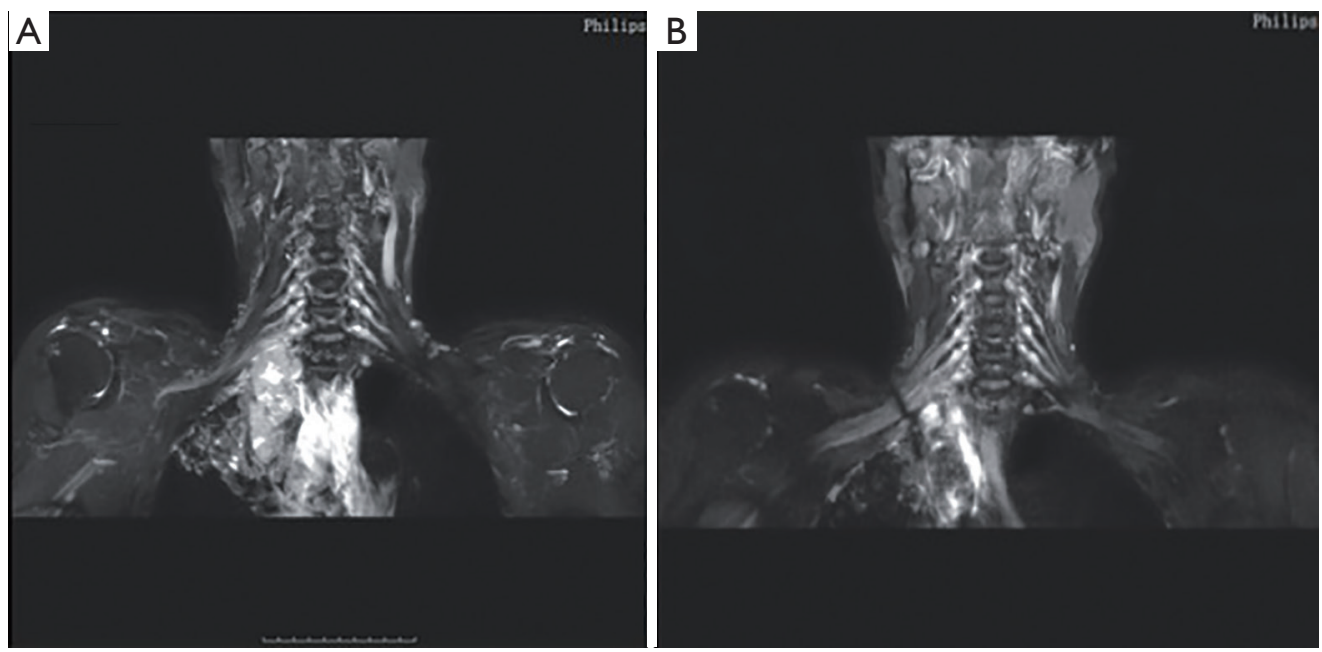

Figure 2 Brachial plexus MRI revealed superior pulmonary sulcus tumor compressing the inferior branch of the brachial plexus. (A) Images before concurrent chemoradiotherapy; (B) images after concurrent chemoradiotherapy.

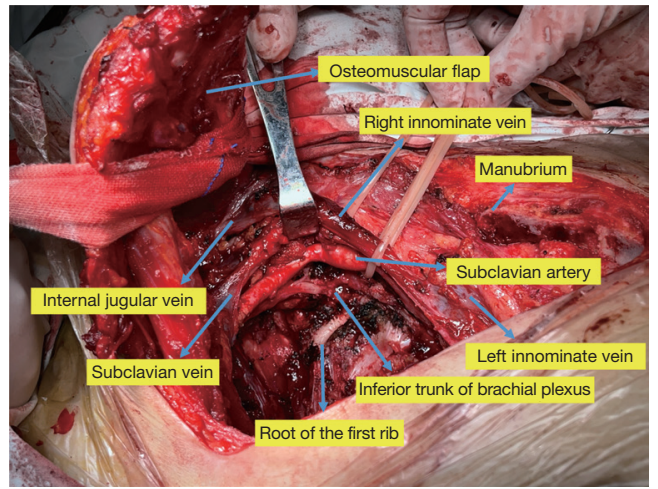

Figure 3 The anatomy of the chest after surgical resection of the tumor and the affected chest wall.

flap, including the clavicle, right half of the manubrium, and the spared pectoralis muscle, was retracted progressively by lace, giving an excellent exposure of the neck and thoracic inlet and upper part of the anterolateral chest wall.

The extension of the tumor to the thoracic inlet of the neck was then carefully assessed. After mobilization of the subclavian vein and its tributaries, the scalenus anterior muscle was divided at its insertion on the first rib, with care being taken not to damage to the phrenic nerve. The subclavian artery and its branches were then dissected, which revealed the tumor resting against the wall of the subclavian artery; the artery was then freed following the subadventitial plane. However, the wall of vertebral artery was invaded, and, since no significant extracranial occlusive disease was detected on preoperative ultrasound evaluation, it was then resected.

The inferior trunk of brachial plexus was compressed but not invaded by the tumor and was isolated from it. Since the upper 2 ribs were invaded by tumor, after mobilization of the posterior part of the upper 2 ribs, they were resected. Resection of the right upper lobe was completed after the tumor was isolated from the apex of chest wall. A total of six stations including three mediastinal stations and ten lymph nodes were removed, the right paratracheal nodes (stations 2R, 4R) and subcarinal lymph nodes (station 7) were removed. The parietal pleura around the hilum was divided, the right upper lobe vein was stapled, and the 2 arteries supplying the upper lobe were transected. The fissure was opened and followed by transection of the right upper lobe bronchus. The tumor was en bloc resected from the affected right upper lobe area of the chest wall. Figure 3 displays the anatomy of the chest after surgical resection of the tumor and affected chest wall. The patient suffered prolonged postoperative air leak and empyema. After continuous chest tube drainage and intrapleural fibrinolytic therapy, the patient recovered after treatment, the chest tubes were removed on postoperative day 48 , and he was discharged on postoperative day 52. Macroscopic pathology showed a tumor size of $4.1 \mathrm{~cm}$, however microscopic pathology showed no viable residue tumor but fibrotic tissue, the tumor had pathological complete response to 
induction treatment, a tumor bed size of $4.1 \mathrm{~cm}$, and no tumor-positive lymph nodes. The patient's final stage was ypT0N0M0. Due to inadequate postoperative recovery, postoperative chemotherapy was abandoned for him. No disease recurrence has been found during the 4 months follow-up. All procedures performed in studies involving human participants were in accordance with the ethical standards of the institutional and/or national research committee(s) and with the Helsinki Declaration (as revised in 2013). Written informed consent was obtained from the patient for publication of this case report and accompanying images. A copy of the written consent is available for review by the editorial office of this journal.

\section{International multidisciplinary team discussion}

\section{Discussion among physicians from Shanghai Chest Hospital}

We conducted multidisciplinary discussion for this case. The 61-year-old male patient was referred for hemoptysis with right arm pain. He had a large mass arising from the right upper lobe invading the apex of chest wall and brachial plexus. Biopsy showed indicated stage cT4N2M0, IIIB adenocarcinoma. The process of diagnosis, preoperative evaluation of resectability, decision-making for surgical resection, and postoperative recovery of the patient are worth discussing.

Radiologist: The patient had an $8.6 \mathrm{~cm} \times 5.1 \mathrm{~cm}$ lung mass invading the apex of the chest wall. For the purpose of confirming the relationship between the mass and the apex of the chest wall, MRI provides better assessment of invasion pleura, subclavian artery, branchial plexus, ribs, and vertebrae. Brachial plexus MRI performed before and after induction treatment showed tumor invasion of the upper 2 ribs, relieved invasion to the subclavian artery and branchial plexus, and no invasion to the vertebrae; MRI is quite useful for assessing the probability of radical resection and deciding the best surgical approach.

Medical oncologist: This patient had a right upper lobe tumor invading the apex structure and was diagnosed with stage cT4N2M0, IIIB lung adenocarcinoma. PET showed increased FDG uptake for ipsilateral hilar and mediastinal lymph nodes (station 2R and 4R). Pathological mediastinal staging was decided upon for this patient. The current standard of care for pancoast tumor is trimodality treatment, induction chemoradiotherapy, including 2 cycles of platinum based chemotherapy, and radiotherapy of more than 45 Gy. Evidence was not very strong for consolidation adjuvant therapy after surgical resection, and the reported ability to deliver adjuvant chemotherapy after resection of a lung cancer has consistently been poor (5). Meanwhile, the patient had inadequate postoperative recovery; therefore, postoperative chemotherapy was abandoned for him.

Thoracic surgeon: Surgical resection of pancoast tumor is technically challenging especially after chemoradiation. Tumor had invaded the inferior trunk of the brachial plexus and the anterior portion of the first 2 ribs, and thus the anterior surgical approach was considered to be suitable for exposure. We decided to use the transmanubrial approach because it preserves the clavicle and provides similar exposure to the thoracic inlet. Because the clavicle was preserved, full shoulder movement was well maintained. The patient suffered postoperative empyema which might have resulted from postoperative persisting air leak. Meticulous air leak management and pressure dressing of the apex of the chest may be beneficial for prevention.

Induction chemoradiotherapy followed by radical surgical resection including a lobectomy along the affected area of the chest wall and thorough mediastinal lymph node dissection is the standard treatment for superior sulcus tumor (5). Multimodality therapy is well tolerated, with an overall treatment-related mortality of only $4.5 \%, 33.7 \%$ of the patients have pathological complete response, and the rate of complete resection is quite high, at around 91.6\% (5).

Preoperative evaluation is important for surgical resection, and compared with $\mathrm{CT}$, the multiplanar nature of MRI provides better assessment of invasion into the pleura, subclavian artery, branchial plexus, ribs, and vertebrae (6). For this patient, MRI performed before and after induction treatment showed tumor invasion of the upper 2 ribs, relieved invasion to the subclavian artery and branchial plexus, and no invasion of the vertebrae. MRI was quite useful for assessing the probability of radical resection and informing the decision to use the transmanubrial approach but not the posterior approach.

Surgical resection of the superior sulcus tumor is challenging because of the narrow space and key structures involved, including the subclavian vessels, brachial plexus, and vertebrae in the thoracic inlet. For patients with pathological complete response, tumor invasion to neighboring structures can be relieved, but the fibrotic change and dense desmoplastic tissue reaction between tumor and apex structures may prohibit the resection. In this case, although the tumor did not invade the subclavian 
artery and brachial plexus, the boundary between them was obscure and difficult to dissect. Both the anterior and posterior approaches and their respective variations can be applied to expose the tumor and the involved structures (3,4,7-10). The anterior approach, proposed by Dartevelle, has been widely accepted; compared with the conventional posterior approach, it provides more radical resection based on better exposure of the subclavian vessel and brachial plexus $(9,10)$. The classical anterior approach is performed by resecting the medial half of the clavicle and the insertions of sternomastoid and major pectoral muscles, which may lead to serious postoperative alterations in shoulder mobility and cervical posture; therefore, we prefer to apply a transmanubrial technique which provides similar exposure to the thoracic inlet but preserves the function of the clavicle and sternomastoid and major pectoral muscles (4). The drawback of the transmanubrial approach is the difficulty in lobectomy, and additional incisions may sometimes be needed for complete resection. We completed the upper lobectomy smoothly without an additional posterior incision. It is easier to begin the lobectomy from the hilum and proceed in order to the vein, artery fissure, and bronchus.

In conclusion, management of superior sulcus tumor is challenging, and the transmanubrial approach can be applied for the resection of tumors invading the branches of the subclavian artery; however, postoperative complications caused by a large defect of the chest should be carefully treated.

\section{The following questions are for international experts}

Question 1: The current standard treatment for pancoast tumor is trimodality therapy, consisting of induction chemoradiotherapy followed by radical surgical resection; however, the evidence for trimodality therapy for pancoast tumor is not very strong. New options including targeted therapy and immune checkpoint inhibitors are being applied as induction treatment for locally advanced lung cancer and have yielded promising outcomes. What is the current evidence and future perspective on these treatments for pancoast tumor?

Seong Yong Park: In the era of precision medicine, the molecular profile of cancer can offer the important clues. Especially this patient was diagnosed as lung adenocarcinoma, so the mutation profile such as EGFR or ALK and PD-L1 expression can be examined before starting the conventional neoadjuvant chemoradiotherapy. Even though classical trimodality therapy is still standard treatment for pancoast tumor, the preoperative target therapy or immunotherapy combined with chemoradiation can be considered. Due to the rarity and aggressiveness of pancoast tumor, the current evidence in the combination of target therapy or immunotherapy with other neoadjuvant regimens is still lacking, and it has to be studied in further trials.

Francesco Grossi: Trimodal treatment remains the standard treatment for pancoast tumor. Ongoing studies of neoadjuvant chemotherapy + immunotherapy are not associated with radiotherapy. The results of the phase II and the preliminary results of the phase III studies in particular of the CheckMate 816 trial are very promising. In this trial the pathological complete response rate is $24 \%$ for nivolumab + chemotherapy compared to $2.2 \%$ for chemotherapy alone with a minor pathological response rate (defined as less than $10 \%$ residual viable tumor cells in both primary tumor and sampled lymph nodes resected) of $36.9 \% \mathrm{vs}$. $8.9 \%$ in favor of immunotherapy. At present there is a lack of comparison studies in pancoast tumor between standard trimodal treatment vs chemotherapy + immunotherapy for this reason we don't know if this strategy is better compared to the standard of treatment with chemoradiotherapy. With regard to molecularly targeted therapies, the results of some studies conducted in patients with EGFR mutation treated with neoadjuvant EGFR TKIs compared to neoadjuvant chemotherapy have given encouraging results but similarly as for immunotherapy we have not data for pancoast tumor.

Question 2: Nodal involvement of pancoast tumor is different from that of classical non-small cell lung cancer. Some studies have shown that for patients with pancoast tumor, ipsilateral supraclavicular nodal involvement-classified as N3 disease involvementmay have prognostic significance more similar to that of $\mathrm{N} 1$ disease. $\mathrm{N} 2$ disease was previously listed as a contraindication for surgical resection, but what are the current procedures recommended for the surgical treatment of pancoast tumor with $\mathbf{N} 2$ disease? What should be considered in the surgical decision-making for patients with different responses to induction treatment?

Seong Yong Park: Current NCCN guideline recommends the surgical resection in cT4N0-1, IIIA pancoast tumor NSCLC. This patient had cT4N2 IIIB cancer, so this patient is out of the classic surgical indication. However, 
after the neoadjuvant therapy, the mediastinal downstaging was observed in chest CT and surgical resection was properly performed. The important parts for deciding the surgical candidates after neoadjuvant therapy in pancoast tumor are nodal status and the resectability; if the patient shows still nodal involvement at the mediastinum (N2 disease), the surgical resection has to be considered cautiously. The decision could be made based on the imaging studies and with invasive nodal staging such as EBUS or mediastinoscopy.

Regarding the nodal status of pancoast tumor, some studies have reported that ipsilateral supraclavicular nodal involvement could be considered as N1 disease in pancoast tumor. These nodes are in close vicinity of the tumor and therefore could have the characteristics of the biological behavior of local nodes (11). However, these studies are from the retrospective studies with small numbers of patients, so the IASLC nodal map has to be applied even in pancoast tumor.

Francesco Grossi: In general, N3 disease is considered an inoperable disease only suitable for chemotherapy + radiotherapy treatment if feasible. There is currently no evidence from comparative studies that a surgical approach after chemoradiotherapy is better than chemoradiotherapy alone. In the international guidelines Pancoasts with $\mathrm{N} 2$ are excluded from surgical treatment however, being standard in these patients chemoradiotherapy as in the neoadjuvant treatment for Pancoast, a re-evaluation of the response after chemoradiotherapy that demonstrate a significant reduction of the disease requires in my opinion a re-evaluation by the disease management team for considering a surgical resection as occurred in the case report described.

\section{Question 3: Various approaches have been used for surgical resection; how do you determine the most suitable surgical approach for each patient? Which approach do you prefer?}

Seong Yong Park: Personally I prefer the anterior approach. In case of invasion to subclavian artery or brachial plexus like this patient, I prefer the anterior Dartevelle approach can provide the good exposure of great vessels and nerve. The Proper managing the great vessels are utmost important for the surgery of pancoast tumor. If the cancer locates nearly the posterior structures such as posterior scalene muscle or posterior part of subclavian and vertebral artery, Shaw-Paulson approach (posterior approaches) could be tried. The dissection of subcarinal node could be challenging during anterior approach, but it is possible with retraction of RUL bronchus stump and bronchus intermedius inferiorly. If the exposure of subclavian vein and artery is not good, the resection of manubrium and clavicle can be also considered. The functional impairment after resection of manubrium and clavicle is not more severe than expect. In case series by Kachala, the functional assessment using the QuickDASH outcome measure revealed minimal loss in upper extremity function after the resection of manubrium and clavicle (12).

Francesco Grossi: From the point of view of the medical oncologist, the best surgical approach is the one that best guarantees a radical resection of the tumor without residual disease with fewer problems for the patient.

\section{Question 4: Classical surgical resection for pancoast} tumor is quite destructive and may compromise the ability to deliver adjuvant chemotherapy after resection. Minimizing surgical trauma may enhance the postoperative recovery and improve the ability of delivering adjuvant treatment. What is your opinion on the future role of minimally invasive surgery including both thoracoscopic and robotic approaches for resection of pancoast tumor?

Seong Yong Park: I think that the classic open resection has a role for the pancoast tumor. By the thoracoscopic or robotic approaches, the resection of first rib is possible, but resection and reconstruction of subclavian vein or artery is technically demanding. In addition, the extent of resection in pancoast tumor is extensive, therefore the surgical trauma could not be reduced even the minimally invasive surgery is applied.

Francesco Grossi: I am in favor of the minimally invasive approach because it reduces hospitalization times and postoperative complications as long as it does not compromise surgical radicality and lymph node dissection. Adjuvant chemotherapy it is not recommended in patients who have had neoadjuvant treatment with chemotherapy and radiotherapy.

Question 5: The patient in this case report suffered postoperative empyema which might have resulted from a large defect of the thoracic cage and prolonged air leak. What can be done perioperatively in this and similar cases to decrease the risk and accelerate the recovery of postoperative empyema?

Seong Yong Park: Reduing the air leakage is very important 
especially extensive lung resection combined with chest wall resection like this patient. The bronchial stump always has to be covered with pericardial fat or pleura and the air leakage point from the lung parenchyma has to be always meticulously sutured. If the empyema develops, the surgical drainage of empyema is usually recommended. If there is the remnant space, this space has to be filled with viable tissue; the muscle flap such as pectoralis major muscle of latissimus dorsi can be considered, and also I have a transposition of the omental flap via substernal route to the apex.

Francesco Grossi: Unfortunately, it is not possible to predict any postoperative complications in most cases. I think that the case described was treated in the best possible way and the complications that occurred after surgical resection were not foreseeable. The radiological response to neoadjuvant therapy oriented towards surgical resection which was successful in light of the pathological complete response obtained.

\section{Acknowledgments}

Funding: None.

\section{Footnote}

Reporting Checklist: The authors have completed the CARE reporting checklist. Available at https://dx.doi. org/10.21037/atm-21-4698

Conflicts of Interest: All authors have completed the ICMJE uniform disclosure form (available at https://dx.doi. org/10.21037/atm-21-4698). The authors have no conflicts of interest to declare.

Ethical Statement: The authors are accountable for all aspects of the work in ensuring that questions related to the accuracy or integrity of any part of the work are appropriately investigated and resolved. All procedures performed in studies involving human participants were in accordance with the ethical standards of the institutional and/or national research committee(s) and with the Helsinki Declaration (as revised in 2013). Written informed consent was obtained from the patient for publication of this case report and accompanying images. A copy of the written consent is available for review by the editorial office of this journal.

Open Access Statement: This is an Open Access article distributed in accordance with the Creative Commons Attribution-NonCommercial-NoDerivs 4.0 International License (CC BY-NC-ND 4.0), which permits the noncommercial replication and distribution of the article with the strict proviso that no changes or edits are made and the original work is properly cited (including links to both the formal publication through the relevant DOI and the license). See: https://creativecommons.org/licenses/by-nc-nd/4.0/.

\section{References}

1. Marulli G, Battistella L, Mammana M, et al. Superior sulcus tumors (Pancoast tumors). Ann Transl Med 2016;4:239.

2. Detterbeck FC. Changes in the treatment of Pancoast tumors. Ann Thorac Surg 2003;75:1990-7.

3. Kratz JR, Woodard G, Jablons DM. Management of Lung Cancer Invading the Superior Sulcus. Thorac Surg Clin 2017;27:149-57.

4. Grunenwald D, Spaggiari L. Transmanubrial osteomuscular sparing approach for apical chest tumors. Ann Thorac Surg 1997;63:563-6.

5. Rusch VW, Giroux DJ, Kraut MJ, et al. Induction chemoradiation and surgical resection for non-small cell lung carcinomas of the superior sulcus: Initial results of Southwest Oncology Group Trial 9416 (Intergroup Trial 0160). J Thorac Cardiovasc Surg 2001;121:472-83.

6. Kichari JR, Hussain SM, Den Hollander JC, et al. MR imaging of the brachial plexus: current imaging sequences, normal findings, and findings in a spectrum of focal lesions with MR-pathologic correlation. Curr Probl Diagn Radiol 2003;32:88-101.

7. Nazari S. Transcervical approach (Dartevelle technique) for resection of lung tumors invading the thoracic inlet, sparing the clavicle. J Thorac Cardiovasc Surg 1996;112:558-60.

8. Kent MS, Bilsky MH, Rusch VW. Resection of superior sulcus tumors (posterior approach). Thorac Surg Clin 2004;14:217-28.

9. Macchiarini P. Resection of superior sulcus carcinomas (anterior approach). Thorac Surg Clin 2004;14:229-40. 
Page 8 of 8

10. Dartevelle PG, Chapelier AR, Macchiarini P, et al. Anterior transcervical-thoracic approach for radical resection of lung tumors invading the thoracic inlet. J Thorac Cardiovasc Surg 1993;105:1025-34.

11. Panagopoulos N, Leivaditis V, Koletsis E, et al. Pancoast
Bao et al. Pancoast tumor resected by the transmanuburial approach

tumors: characteristics and preoperative assessment. J Thorac Dis 2014;6 Suppl 1:S108-15.

12. Kachala SS, D'Souza DM, Teixeira-Johnson L, et al. Surgical Management of Sternoclavicular Joint Infections. Ann Thorac Surg 2016;101:2155-60.

Cite this article as: Bao F, Yu F, Hao X, Gu Z, Park SY, Grossi F, Fang W. Surgical resection of superior pulmonary sulcus tumor after neoadjuvant chemoradiation via the anterior transmanubrial approach: a case report. Ann Transl Med 2021;9(20):1603. doi: 10.21037/atm-21-4698 